\title{
A novel mutation of the COLQ gene causes congenital myasthenic syndromes: A case report
}

\author{
*Shiyi Yang $P h D, *$ Jing Luo $M D$, Fei Xiao $M D$ \\ *SY Yang and J Luo contributed equally to the paper and are co-first author.
}

Department of Neurology, The First Affiliated Hospital of Chongqing Medical University, Chongqing Key Laboratory of Neurology, Chongqing, China

\begin{abstract}
Congenital myasthenic syndromes (CMS) are a group of heterogeneous disorders of the neuromuscular junctions, characterised by fluctuating and fatigable weakness with an early onset. Endplate acetylcholinesterase deficiency (EAD) due to mutations in COLQ is a subtype of CMS whose key clue for diagnosis is repetitive compound muscle action potential (R-CMAP) under nerve conduction studies. In light of the significant overlap of clinical symptoms, misdiagnosis is common for CMS, causing delayed or incorrect treatments. Here, we report a case of CMS due to a novel mutation in COLQ with a typical R-CMAP and discuss the significance of R-CMAP for diagnosis.
\end{abstract}

Keywords: Congenital myasthenic syndromes, COLQ, endplate acetylcholinesterase deficiency

\section{INTRODUCTION}

Congenital myasthenic syndromes (CMS) are a group of rare heterogeneous disorders of the neuromuscular junctions (NMJs). Typically, these conditions are due to dysfunctional proteins located in the presynaptic terminal, synaptic cleft, postsynaptic terminal, or proteins affecting the functions of NMJs. ${ }^{1}$ CMS are characterised by abnormal fatigue and fluctuating weakness involving the ocular, facial, respiratory, bulbar, and limb muscles. To date, 32 genes have been identified as responsible for CMS. Endplate acetylcholinesterase deficiency (EAD) due to mutations in COLQ is the third most frequent type of CMS subtype. ${ }^{2}$ This is an autosomal recessive inherited CMS caused by a lack of acetylcholinesterase in NMJs. Herein, we report a case of EAD with a novel mutation in COLQ, which was previously misdiagnosed as myasthenia gravis (MG).

\section{CASE REPORT}

A 9-year-old boy had impaired eye movement since he was 7 months old. He started to walk at 18 months of age, followed by fluctuating generalised weakness and fatigue. When he was 4 years old, he was diagnosed with MG due to repetitive nerve stimulation (RNS) with low-frequency stimuli of the bilateral facial nerves, and the right median nerve also showed a significant decrease. He received pyridostigmine as treatment; however, the treatment was ineffective, so he took it only intermittently. His weakness had progressed significantly in the last two years, and he was unable to walk for 100 meters. He restarted treatment with pyridostigmine, which aggravated his symptoms. The patient did not complain of diplopia, dysphagia, or paraesthesia. His intelligence was normal. There was no family or birth delivery difficulty history of note.

Neurological examination revealed mild bilateral ptosis, bilateral ophthalmoplegia in both the horizontal and vertical directions, and symmetric weakness of the neck muscle (4/5), biceps (3/5), deltoid (3/5), and distal muscles (515), according to the Medical Research Council (MRC) scale. His muscle tone and tendon reflexes were mildly diminished. The remaining examinations were unremarkable.

Laboratory examinations, including creatine kinase, serum lactic acid, anti-acetylcholine receptor (anti-AChR), and anti-muscle-specific tyrosine kinase (anti-MuSK) antibodies, were all normal. Cranial magnetic resonance imaging, and muscle biopsy of the left biceps were all normal. The sensory and motor nerve conduction studies (NCS) and electromyography were normal; while a single stimulus elicited repetitive compound muscle action potentials (R-CMAPs) in the

Address correspondence to: Fei Xiao, M.D., Department of Neurology, The First Affiliated Hospital of Chongqing Medical University, Chongqing Key Laboratory of Neurology, 1st You Yi Road, 400016, Chongqing, China. Tel: 8623 89012009, email: feixiao81@ 126.com

Date of Submission: 13 June 2021; Date of Acceptance: 13 August 2021

https://doi.org/10.54029/2021mue 
peroneal and ulnar nerves (Figure 1. a, b). Slow repetitive stimulation $(3 \mathrm{~Hz})$ of the right median nerve revealed reproducible CMAP decrement $(>10 \%)$ and rapid repetitive stimulation $(30 \mathrm{~Hz})$ of the right tibial nerve revealed reproducible CMAP decrement, measured at 48\% (Figure 1. $\mathrm{c}, \mathrm{d})$.

Based on the diminished muscle tone, tendon reflex, and negative pathological reflexes, we localised the lesion to the lower motor neuron.
The possible topographies were motor neurons in the anterior horn, peripheral nerve, neuromuscular junction (NMJ), and the muscle. As there was no muscular dystrophy or paraesthesia, and NCS was normal, neurogenic disorders appear less likely; whilst extraocular muscle involvement and ptosis can be present in some genetic myopathies, such as mitochondrial myopathy, congenital myopathy, and myotonic myopathy. In this patient, the serum $\mathrm{CK}$ levels were within the normal range, and EMG
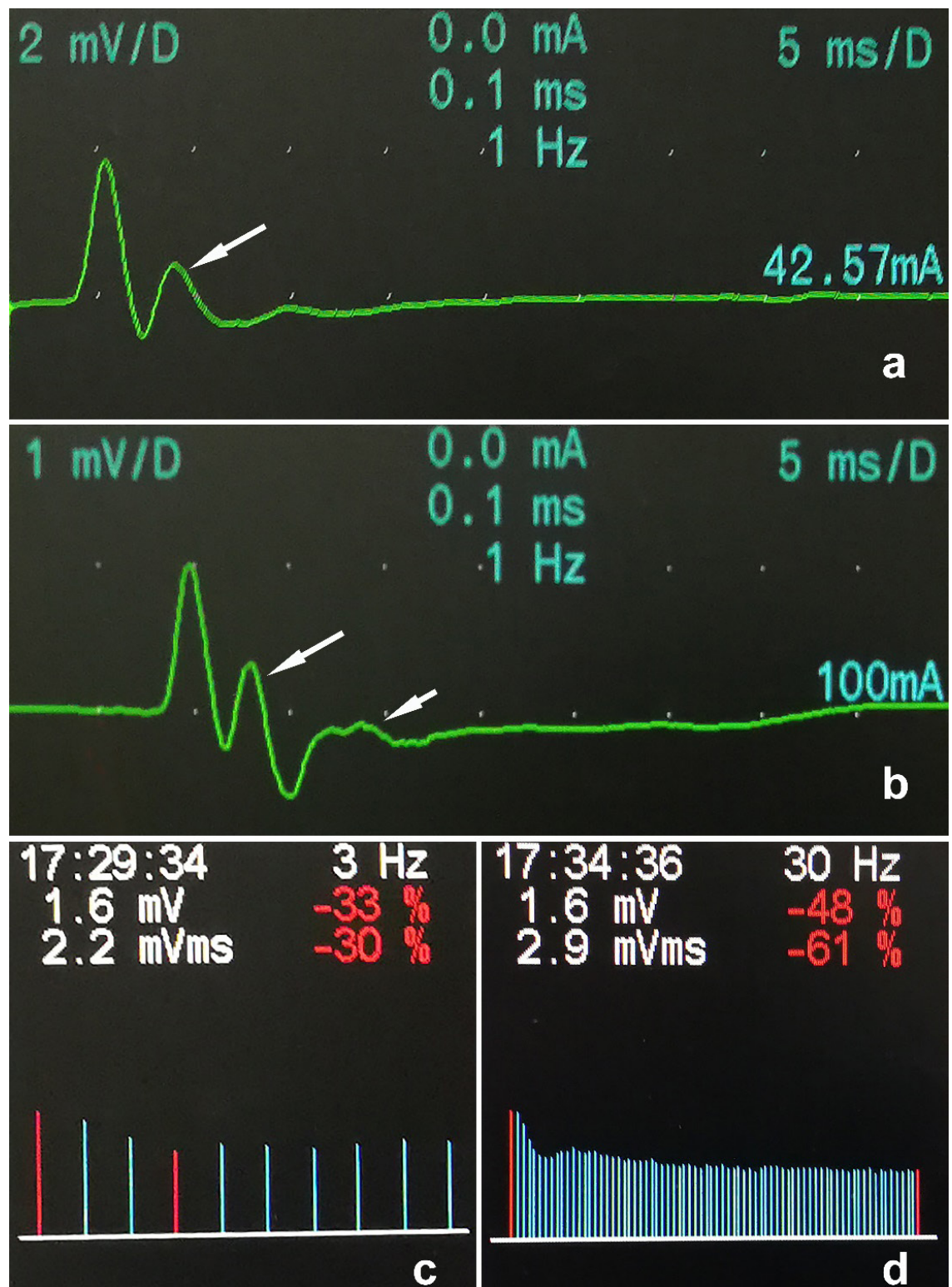

Figure 1. Single stimulus elicited repetitive compound muscle action potential (R-CMAP) in the left peroneal nerve (a) and right ulnar nerve (b). White arrows indicate R-CMAP. Slow repetitive stimulation $(3 \mathrm{~Hz})$ of the right median nerve reveals reproducible CMAP decrements, measured at $33 \%$ (c) and rapid repetitive stimulation $(30 \mathrm{~Hz})$ of the right tibial nerve reveals reproducible CMAP decrements, measured at $48 \%$ (d). 
and muscle biopsy findings were unremarkable; thus, muscular disorders seemed unlikely. Fatigable weakness, fluctuating symptoms, and decremental RNS responses indicated NMJ disorders. MG is the most common NMJ disorder. However, patients who are double-seronegative for anti-AChR and anti-MuSK antibodies, as in the present case, account for less than $20 \%$ of MG cases. ${ }^{3}$ The aggravation of symptoms with acetylcholinesterase inhibitors (AChEIs) prompted us to rule out MG. Onset at infancy and delay in motor development suggested congenital diseases; thus, CMS seemed to be the most likely diagnosis.

AChEI is effective for some types of CMS; however, it can be ineffective or even aggravate symptoms in other types of CMS due to the different underlying pathological mechanisms. Among certain subtypes that do not respond to AChEI, R-CAMP is an important feature of two subtypes: slow-channel congenital myasthenic syndrome (SCCMS) and EAD due to mutations in COLQ. ${ }^{1}$ Therefore, the patient underwent genetic testing, which revealed compound heterozygous mutations in the COLQ gene, a missense mutation c. $175 \mathrm{C}>\mathrm{T}$ from his father, and a null mutation c. $538 \mathrm{G}>\mathrm{T}$ from his mother. Thus, the diagnosis of EAD caused by COLQ mutations was established. Albuterol was then administered to the patient at a dose of $2 \mathrm{mg}$ twice daily, and his weakness was significantly relieved after 3 months.

\section{DISCUSSION}

Herein, we report a case of EAD and identified a novel mutation in COLQ. EAD caused by mutations in COLQ, which encodes the triplestranded collagenic tail responsible for anchoring acetylcholinesterase to the synaptic basal lamina, is a type of synaptic basal lamina-associated CMS. Acetylcholinesterase is absent in the postsynaptic terminal, and structural abnormalities of NMJs have been previously identified. ${ }^{4}$ Onset of EAD stretches from birth to early childhood. Muscle weakness is typically generalised. Involvement of the ocular muscles, delayed motor milestones, respiratory insufficiency, and scoliosis are common $^{5}$, and a slow pupillary reaction to light has also been noted in some patients. ${ }^{1}$ Given the absence of acetylcholinesterase, AChEI is ineffective, it can even worsen the symptoms. The resulting prolonged endplate potential (EPP) leads to the appearance of R-CAMP, which is a key clue for the diagnosis. Ephedrine and albuterol are however, effective. ${ }^{1}$

It has been said that more than $80 \%$ of children with $\mathrm{CMS}^{6}$ and $94 \%$ of adult patients ${ }^{7}$ are misdiagnosed; and seronegative $\mathrm{MG}$ is one of the most common misdiagnoses in clinical practice. ${ }^{6,7}$ In conclusion, CMS should be considered if a patient with MG-like symptoms has the following: 1) onset in infancy or early childhood, 2) all the autoimmune antibodies of MG could not be detected 3) AChEI was ineffective, and 4) R-CAMP was found.

\section{ACKNOWLEDGEMENT}

This work is supported by the Fifth Senior Medical Talents Program of Chongqing for Young and Middle-aged, and medical research project of Chongqing Science and Technology Commission and Health Commission (No.2019MSXM075).

\section{DISCLOSURE}

A written informed consent was obtained from the patient's family. Ethical approval for publishing this case report has been ethical committee of The First Affiliated Hospital of Chongqing Medical University.

Financial support: This study was funded by the Fifth Senior Medical Talents Program of Chongqing for Young and Middle-aged, and medical research project of Chongqing Science and Technology Commission and Health Commission (No.2019MSXM075).

Conflict of interest: None

\section{REFERENCES}

1. Engel AG,Shen XM,Selcen D, Sine SM. Congenital myasthenic syndromes: pathogenesis, diagnosis, and treatment. Lancet Neurol 2015;14(5):461.

2. Finsterer J. Congenital myasthenic syndromes. Orphanet J Rare Dis 2019;14(1):57.

3. Gilhus NE, Tzartos S, Evoli A, Palace J, Burns TM, Verschuuren J. Myasthenia gravis. Nat Rev Dis Primers 2019;5(1):30.

4. EngelAG,LambertEH, GomezMR.Anew myasthenic syndrome with end-plate acetylcholinesterase deficiency, small nerve terminals, and reduced acetylcholine release. Ann Neurol 1977;1(4):315-30.

5. Mihaylova V,Müller JS,Vilchez JJ, et al. Clinical and molecular genetic findings in COLQ-mutant congenital myasthenic syndromes. Brain 2008;131(Pt 3):747-59.

6. Kinali M, Beeson D, Pitt M, et al. Congenital myasthenic syndromes in childhood: diagnostic and management challenges. J Neuroimmunol 2008;201202:6-12.

7. Kao JC, Milone M, Selcen D, Shen XM, Engel AG, Liewluck T. Congenital myasthenic syndromes in adult neurology clinic: A long road to diagnosis and therapy. Neurology 2018;91(19):e1770-e7. 